\title{
Energetics, structure, mechanical and vibrational properties of single-walled carbon nanotubes
}

\author{
Guanghua Gao, Tahir Çağın $\dagger$ and William A Goddard III
}

Materials and Process Simulation Center, Beckman Institute (139-74), Division of Chemistry and Chemical Engineering, California Institute of Technology, Pasadena, CA 91125, USA

Received 6 January 1998

\begin{abstract}
In this paper, we present extensive molecular mechanics and molecular dynamics studies on the energy, structure, mechanical and vibrational properties of single-wall carbon nanotubes. In our study we employed an accurate interaction potential derived from quantum mechanics. We explored the stability domains of circular and collapsed cross section structures of armchair $(n, n)$, zigzag $(n, 0)$, and chiral $(2 n, n)$ isolated single-walled carbon nanotubes (SWNTs) up to a circular cross section radius of $170 \AA$. We have found three different stability regions based on circular cross section radius. Below $10 \AA$ radius only the circular cross section tubules are stable. Between 10 and $30 \AA$ both circular and collapsed forms are possible, however, the circular cross section SWNTs are energetically favorable. Beyond $30 \AA$ (crossover radius) the collapsed form becomes favorable for all three types of SWNTs. We report the behavior of the SWNTs with radii close to the crossover radius $((45,45),(80,0),(70,35))$ under uniaxial compressive and tensile loads. Using classical thin-plane approximation and variation of strain energy as a function of curvature, we calculated the bending modulus of the SWNTs. The calculated bending moduli are $\kappa_{(n, n)}=963.44 \mathrm{GPa}, \kappa_{(n, 0)}=911.64 \mathrm{GPa}$, and $\kappa_{(2 n, n)}=935.48 \mathrm{GPa}$. We also calculated the interlayer spacing between the opposite sides of the tubes and found $d_{(n, n)}=3.38 \AA, d_{(2 n, n)}=3.39 \AA$, and $d_{(n, 0)}=3.41 \AA$. Using an enthalpy optimization method, we have determined the crystal structure and Young's modulus of $(10,10)$ armchair, $(17,0)$ zigzag and $(12,6)$ chiral forms (which have similar diameter as $(10,10)$ ). They all pack in a triangular pattern in two dimensions. Calculated lattice parameters are $a_{(10,10)}=16.78 \AA$, $a_{(17,0)}=16.52 \AA$ and $a_{(12,6)}=16.52 \AA$. Using the second derivatives of potential we calculated Young's modulus along the tube axis and found $Y_{(10,10)}=640.30 \mathrm{GPa}$, $Y_{(17,0)}=648.43 \mathrm{GPa}$, and $Y_{(12,6)}=673.94 \mathrm{GPa}$. Using the optimized structures of $(10,10),(12,6)$ and $(17,0)$, we determined the vibrational modes and frequencies. Here, we report the highest in-plane mode, compression mode, breathing mode, shearing mode and relevant cyclop mode frequencies.
\end{abstract}

M This article features multimedia enhancements available from the abstract page in the online journal; see http://www.iop.org.

\section{Introduction}

Carbon nanotubes were discovered in 1991 by Iijima of NEC corporation [1]. Since then, efforts in synthesis, characterization and theoretical investigation on nanotubes have grown exponentially. This is mostly due to their perceived novel mechanical and electronic properties and their tremendous potential for future technological applications. In 1993, the simplest kind of carbon nanotubes, single-walled carbon nanotubes (SWNTs), were discovered independently by the Iijima group [2] and an IBM team headed by Bethune [3]. These SWNTs can be regarded as a rolled-up graphite sheet in cylindrical form.

$\dagger$ Author to whom all correspondence to be addressed.
Some specific defect-free forms of these SWNTs show remarkable mechanical properties and metallic behavior [4]. These materials present tremendous potential as components for use in nano-electronic and nano-mechanical device applications or as structural elements in various devices.

Thess and co-workers [4] later produced crystalline 'ropes' of metallic carbon nanotubes with 100-500 SWNTs bundled into a two-dimensional triangular lattice. These tightly bundled linear 'ropes' are expected to have remarkable mechanical properties, as well as superior electronic and magnetic properties. Various levels of studies were performed on the properties of the SWNTs, including use of classical molecular mechanics (MM), 
lattice dynamics (LD), molecular dynamics (MD), tight binding and $a b$ initio level quantum mechanical (QM) methods [5-18]. Robertson et al carried out first studies on the structure and energetics of isolated small-diameter SWNTs [13]. More recent theoretical studies include, behavior of nanotubes under high-rate tensile strain and fracture [14], size-dependent resonance Raman scattering [15] in nanotubes, mechanical behavior of SWNT under compression using MD [16], and a pioneering study on the structural flexibility of carbon nanotubes using HREM and atomistic simulations addressing the role of van der Waals radii reported by Tersoff and co-workers $[17,18]$.

In this paper, we present a detailed study of the energetics, structures, and mechanical properties of SWNT with different radius and chirality (armchair $(n, n)$, chiral $(2 n, n)$, and zigzag $(n, 0))$. We used an accurate force field, derived through QM calculations, to represent the interactions between the carbon atoms [19]. These interaction potentials were used earlier in studying structure, mechanical and vibrational properties of graphite, various fullerenes and intercalated compounds of fullerenes [20] and nanotubes [21]. In our studies, we employed classical MD and molecular mechanics methods as implemented in MPSim, a massively parallel program for materials simulations [22]. MD runs are performed to anneal the structures, whereas molecular mechanics, energy and/or enthalpy minimization, are applied at the end of the annealing cycle to obtain the final optimized structures. Using the analytical second derivatives of the potential energy, we also calculate the vibrational modes and frequencies of three kinds of nanotube bundles, $(10,10)$ arm chair, $(17,0)$ zigzag and $(12,6)$ chiral. These tubes have comparable cross section diameters, and are among the easiest to make.

\section{Energetics and the stability of circular versus collapsed tubes}

In order to assess mechanical stability of various SWNTs, we created three chiral forms $((n, n)$ armchair, $(n, 0)$ zigzag, and $(2 n, n))$ with various diameters. For each form, we studied two sets of initial structures, (1) perfect circular cross section and (2) elongated or collapsed cross section. For the collapsed structures, the opposite walls in the middle section are within van der Waals distance and the shape at the two ends is close to circular with a diameter of $D$ 10.7 ̊. To mimic a long isolated nanotube, we imposed a periodic boundary condition along the $c$-axis (the tube direction). To eliminate the intertube interactions, we set the cell parameters $a$ and $b$ to 50 times the circular tube diameter. Energy and structural optimizations were carried out using MPSim. Figure 1 shows the strain energy per carbon atom versus the radius of its circular form. We put the two sets (collapsed and circular) with three chiral forms $((n, n)$ armchair, $(n, 0)$ zigzag, and $(2 n, n))$ on the same plot. For all three forms, there are regions associated with two transition radii $\left(R_{1}\right.$ and $\left.R_{2}\right)$. For tubes with 'circular' radius smaller than $R_{1}$, only the circular form is possible, the collapsed initial structure transformed to the circular form during the structural optimization. For

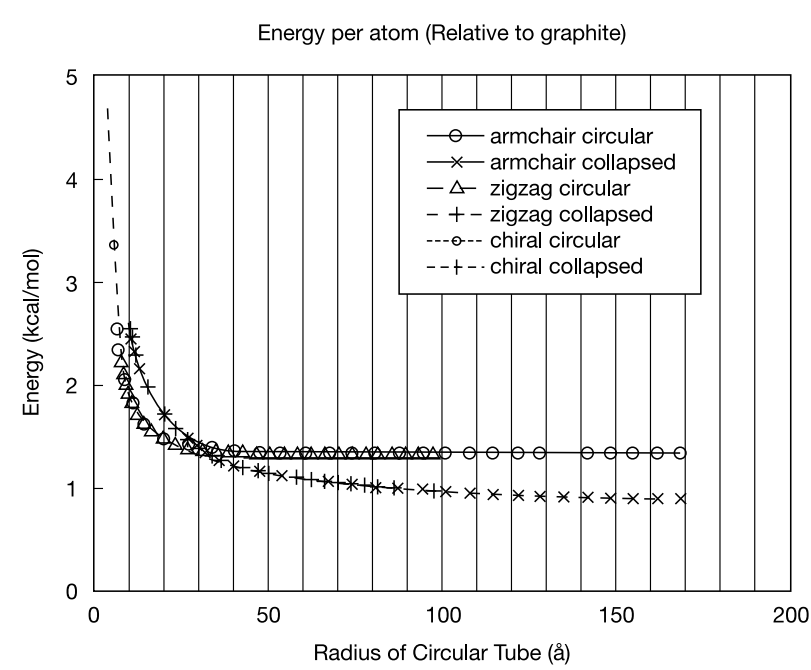

Figure 1. Energy per carbon atom (measured relative to graphite) of stable structures for $(n, n)$ armchair, $(2 n, n)$ chiral, and $(n, 0)$ zigzag SWNTs; $R$ is the radius of the corresponding 'circular' form.

tubes with 'circular' radius between $R_{1}$ and $R_{2}$, there are two stable structures with the circular structure energetically more stable. For tubes with 'circular' radius larger than $R_{2}$, the collapsed form becomes energetically favored while the circular form becomes metastable. The structures and radii of the first transition are:

- $(n, n)$ armchair: $R_{1}$ is between $10.77 \AA$ of $(16,16)$ and $11.44 \AA$ of $(17,17)$;

- $(2 n, n)$ chiral: $R_{1}$ is between $10.28 \AA$ of $(20,10)$ and $11.31 \AA$ of $(22,11)$;

- $(n, 0)$ zigzag: $R_{1}$ is between $10.49 \AA$ of $(27,0)$ and $10.88 \AA$ of $(28,0)$.

The structures and the radii of the second transition are:

- $(n, n)$ armchair: $R_{2}$ is between $29.62 \AA$ of $(45,45)$ and $30.30 \AA$ of $(46,46)$;

- $(2 n, n)$ chiral: $R_{2}$ is between $29.82 \AA$ of $(58,29)$ and $30.85 \AA$ of $(60,30)$;

- $(n, 0)$ zigzag: $R_{2}$ is between $29.93 \AA$ of $(77,0)$ and $30.32 \AA$ of $(78,0)$.

Looking at the collapsed structures of various radii along tube axis, we found that they all have two circular (or elliptical) ends with a diameter $D$ of $\tilde{1} 0.5 \AA$ and a flat middle section. The inner wall distances in the flat region are close to $3.4 \AA$, which is very similar to the interlayer spacing of adjacent sheets in graphite crystals. The end sections are highly strained compared with the circular form, thus cost energy. In addition to zero strain energy, the flat region is further stabilized by interlayer van der Waals attractions. The relative strength of these two opposite forces dictates the two structural transformations. Shown in figure 2 are the optimized structures of armchair $(n, n)$ tubes with collapsed initial structures viewed along the $c$-axis. The zigzag $(n, 0)$ and chiral $(2 n, n)$ tubes are similar.

Based on the optimized structures and their energies of the circular form, we can model the basic energetics by approximating the tube as a membrane with a curvature $1 / R$ and bending modulus of $\kappa$ [23]. Assuming $a$ as the 


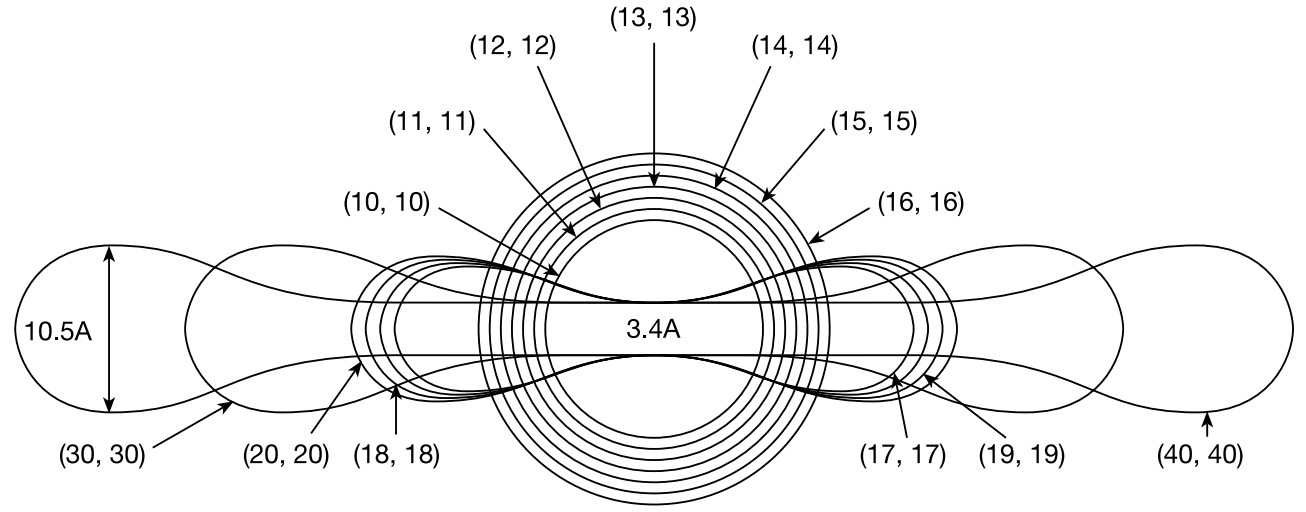

Figure 2. Cross sections of the optimum $(n, n)$ tubes (started from the collapsed forms shown).

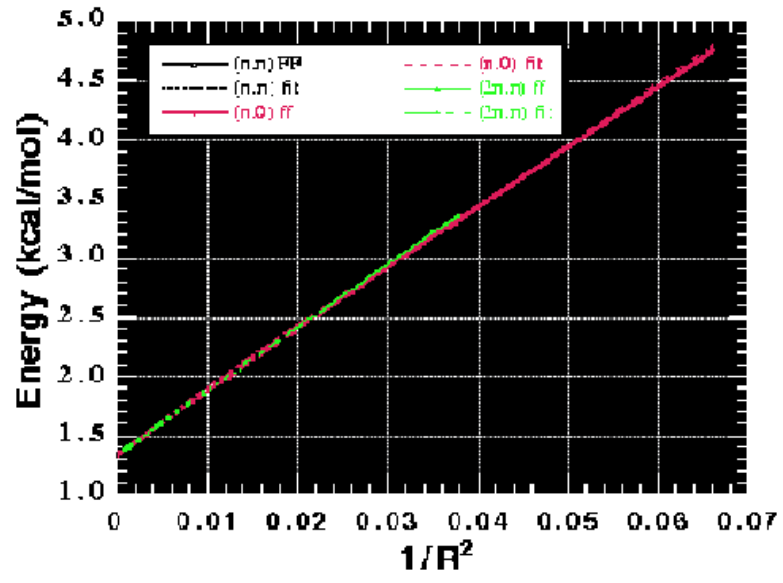

Figure 3. Strain energy as a function of tube radius.

thickness of tube wall, the elastic energy stored in a slab of width $L$, is given by

$$
E_{\text {strain }}=\frac{\pi \kappa L a^{2}}{12 R}
$$

The per atom energy can be written as

$$
E_{C}=\frac{\pi \kappa L a^{2}}{12 R N}+E_{0}
$$

where $N$ is the number of carbon atoms per slab and $E_{0}$ is the energy per carbon atom for tubes as $1 / R$ goes to 0 , i.e. flat sheets. Considering $N=2 \pi \rho L R$ the number of carbon atoms per unit area of tube wall, we have

$$
E_{C}=\kappa \frac{a^{2}}{24 \rho} \frac{1}{R^{2}}+E_{0} .
$$

Setting $a=3.335 \AA$, the same as the spacing between two graphite sheets, $R_{0}=1.410 \AA$, the $\mathrm{C}-\mathrm{C}$ bond distance, we obtained $\kappa_{(n, n)}=963.44 \mathrm{GPa}, \kappa_{(n, 0)}=911.64 \mathrm{GPa}$, and $\kappa_{(2 n, n)}=935.48 \mathrm{GPa}$. These results are plotted against the theoretical estimates in figure 3 .

The bending moduli of sheets with different chirality suggest that the transition radius depends on the chirality,
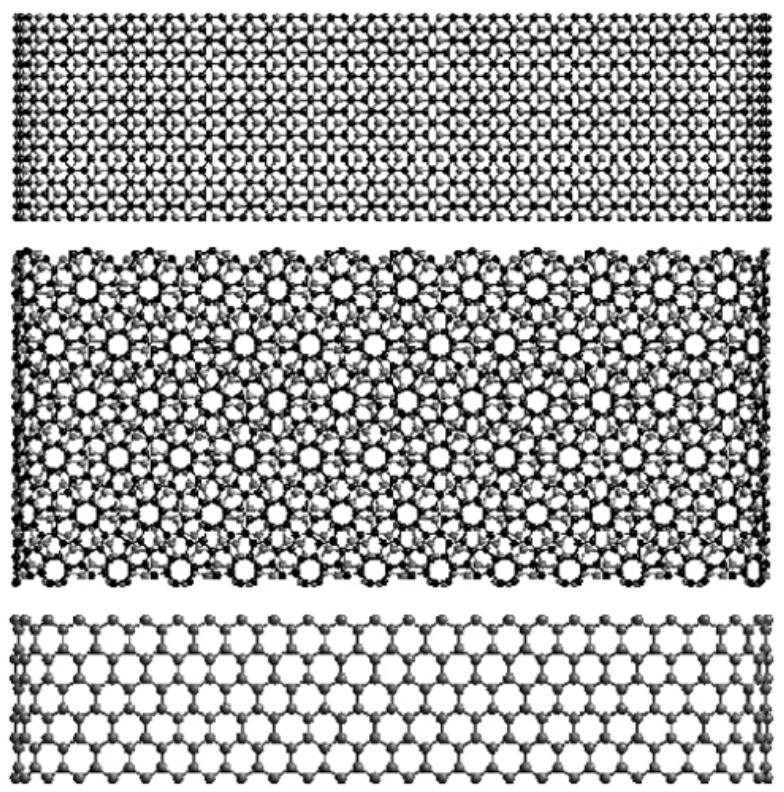

Figure 4. Side views of interlayer stacking patterns of: (a) $(30,30)$ armchair; $(b)(50,25)$ chiral and $(c)(50,0)$ zigzag SWNTs.

with a $(2 n, n)$ transition radius larger than that of $(n, 0)$ zigzag, but smaller than that of $(n, n)$ armchair. That is what we expected, because the higher the bending modulus, the higher the strain energy. The $(n, n)$ armchair has higher binding energy than that of $(2 n, n)$, while the $(n, 0)$ zigzag has lower binding energy than that of $(2 n, n)$. However, by examining the collapsed structures closely, we also found different interlayer stacking in the collapsed region. Figure 4 is the side views of the two attracting layers for three cases. The interlayer stacking patterns are different due to different chirality.

The interlayer distances also differ slightly, with $d_{(n, n)}=3.38 \AA, d_{(2 n, n)}=3.39 \AA$, and $d_{(n, 0)}=3.41 \AA$. Energetically, interlayer attraction of armchair is the best with a per atom energy $E=0.7336 \mathrm{kcal} \mathrm{mol}^{-1}$, and stacking of the opposite walls is almost identical to the graphite stacking. The interlayer attraction in the zigzag form is the worst with a per atom energy $E=$ $0.7439 \mathrm{kcal} \mathrm{mol}^{-1}$, since the carbon atoms on different layers are lined up on top of each other. The attraction 

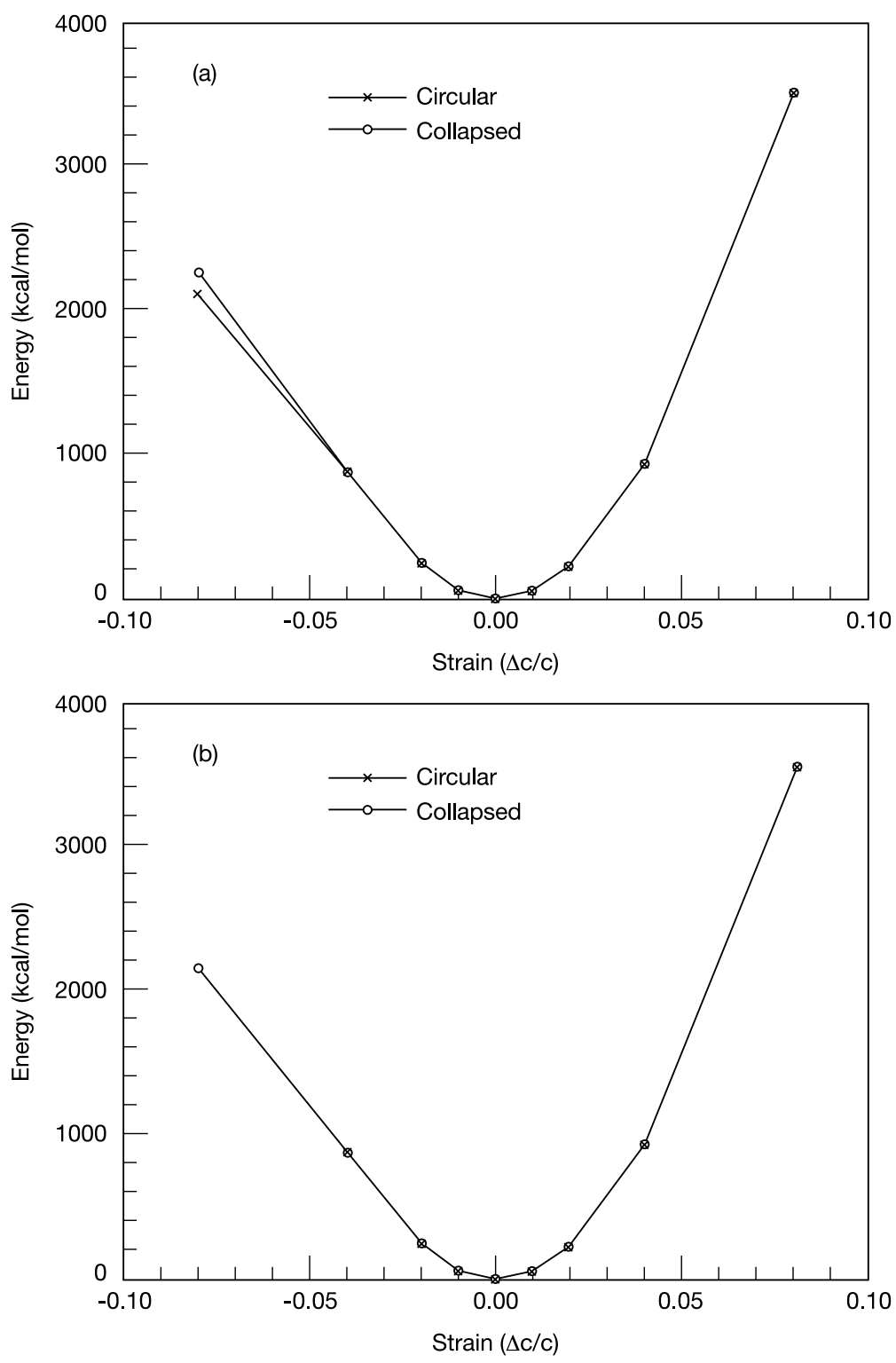

Figure 5. Strain energies, (a) strain energy versus strain for $(45,45)$ armchair SWNT; $(b)$ strain energy versus strain for $(80,0)$ zigzag SWNT; $(c)$ strain energy versus strain for $(70,35)$ chiral SWNT.

energy per atom for the collapsed $(2 n, n)$ chiral nanotube is in between the two. Overall, the two factors (bending modulus and van der Waals attraction) cancel out for different chirality, so that in terms of transition radius and the crossover radius, the size of the circular tube (radius) is the dominant factor in deciding the stable forms.

\section{Stretching and compressing tubes at the crossover radius}

In order to assess the tensile and compressive strength of the SWNTs, we also performed a series of compressive and tensile loading experiments by varying the $c$ lattice parameter, along the tube axis. With respect to the optimum $c$ lattice parameter, $c_{0}$, the strain can be defined as

$$
\epsilon=\frac{\Delta c}{c}
$$

At the crossover radius, we calculated the energies of collapsed and circular tubes of $(45,45),(80,0)$, and $(70,35)$ under compression and tensions along tube axis. In figure $5(a)$, the armchair, figure $5(b)$, the zigzag, and figure $5(c)$, the chiral forms, we observe a nonlinear dependence on the strain.

The tubes are softer under compression due to the buckling effect. We present views along the tube axis and perpendicular to the tube axis in figure 6. Based on these energy versus strain curves, we also calculated 'elastic constants', the second derivative of energy with respect to applied strain, $\epsilon$, along the tube axis, table 1 .

\section{Structure and mechanical properties of packed SWNT crystals}

Among various conformations, the $(10,10)$ armchair SWNT is the easiest to make. We studied the mechanical 


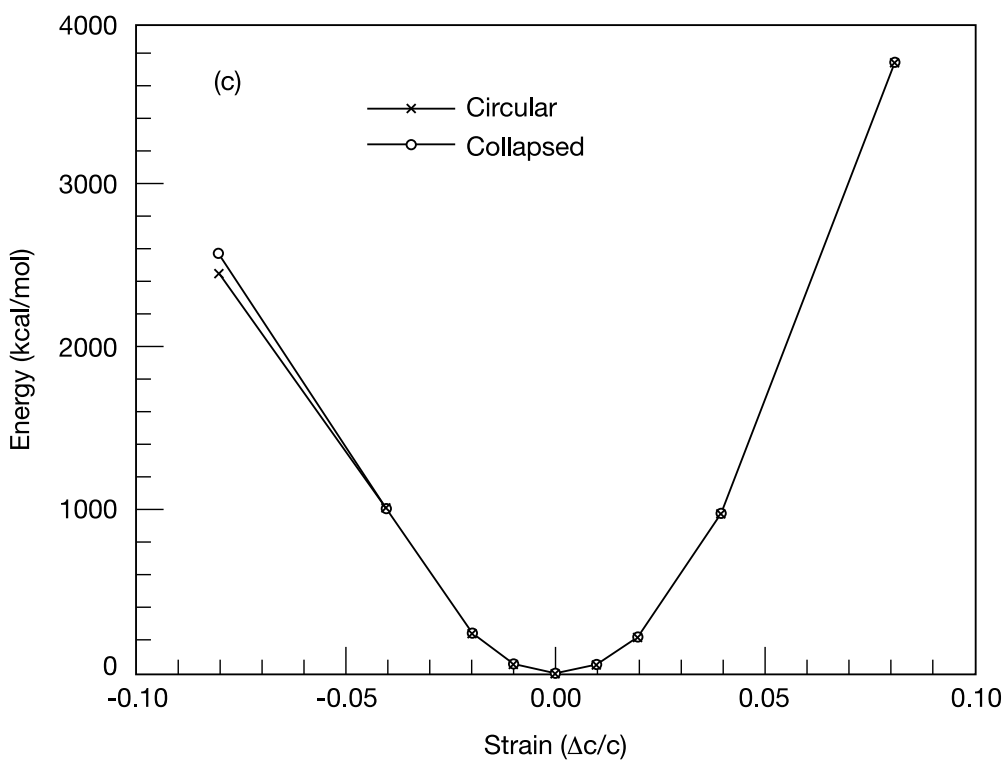

Figure 5. (Continued)

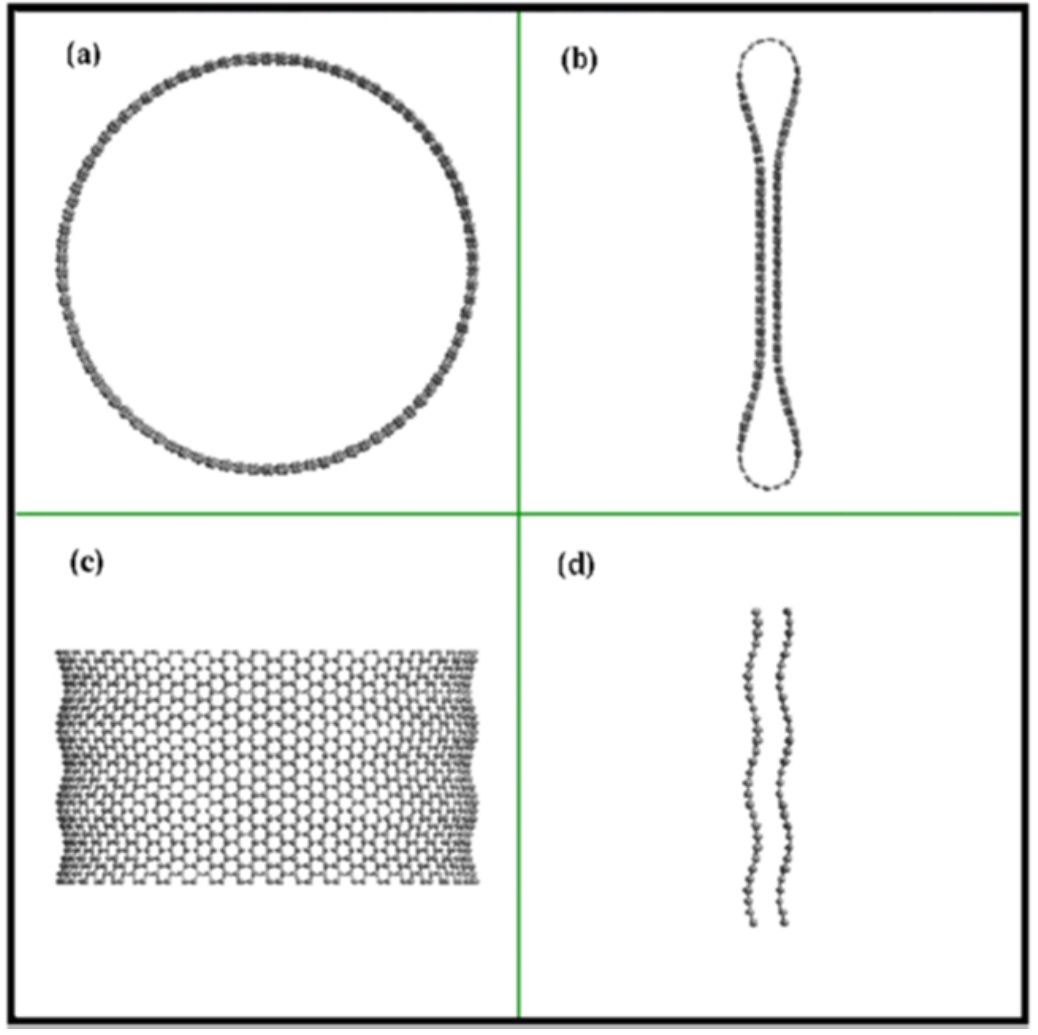

Figure 6. Views of tubes perpendicular to and along tube axis. 

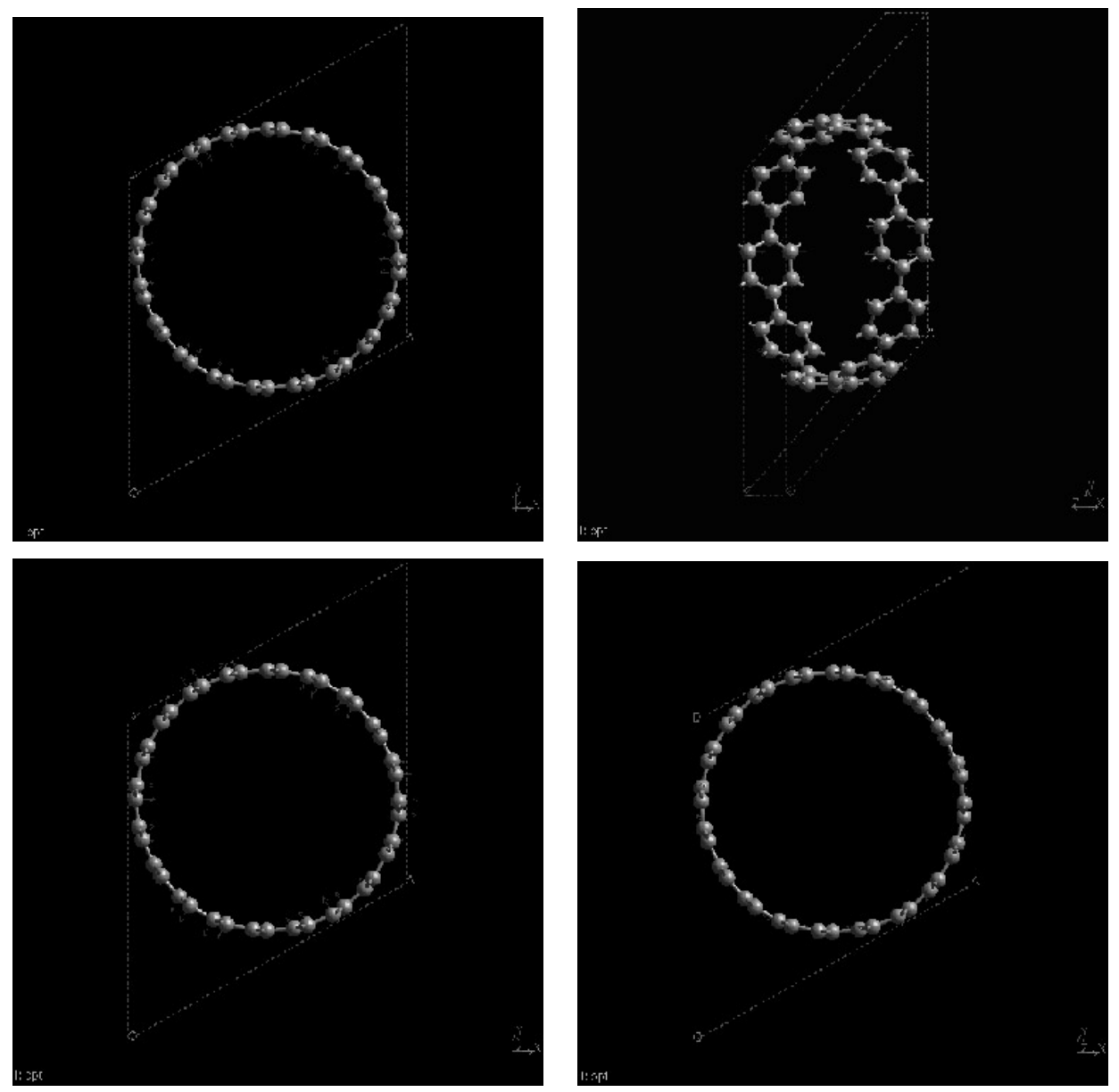

Figure 7. Selected vibrational modes for $(10,10)$ tube, $(a)$ Top left, uniform compression mode; $(b)$ bottom left, shearing along tube mode; $(c)$ top right, breathing mode $(d)$ bottom right, cyclop mode.

M An MPEG movie of this figure is available from the article's abstract page in the online journal; see http://www.iop.org.

Table 2. The uniform compression (first row) and the highest in plane modes (second row) for $(10,10)$ SWNT.

\begin{tabular}{rrrrrcccc}
\hline$(7,7)$ & $(8,8)$ & $(9,9)$ & $(12,6)$ & $(17,0)$ & $(10,10)$ & $(11,11)$ & $(12,12)$ & $(13,13)$ \\
\hline 261 & 231 & 207 & 202 & 188 & 186 & 168 & 152 & 138 \\
1583 & 1584 & 1584 & 1585 & 1586 & 1584 & 1584 & 1584 & 1584 \\
\hline
\end{tabular}

Table 1. Elastic modulus along the tube axis for circular and collapsed forms expressed in units of $10^{5} \mathrm{kcal} \mathrm{mol}^{-1}$.

\begin{tabular}{llll}
\hline & $(45,45)$ & $(80,0)$ & $(70,35)$ \\
\hline Circular & 6.2162 & 6.3965 & 6.7431 \\
Collapsed & 5.7351 & 5.9747 & 6.7105 \\
\hline
\end{tabular}

properties of its bulk phase $(10,10)$ tube bundles. We also calculated the bulk properties of $(17,0)$ zigzag and $(12,6)$ chiral tubes, with cross section radii close to that of $(10,10)$ armchair tube. In the bulk phase we determine the specific packing, density, lattice parameters. MD and MM studies led to a triangular packing as the most stable form for all three forms. The triangular lattice parameter for $(10,10)$ armchair is $a=16.78 \AA$ with a density $\rho=1.33 \mathrm{~g} \mathrm{~cm}^{-3}$. For the $(17,0)$ zigzag, they are $a=16.52 \AA$ and $\rho=1.34 \mathrm{~g} \mathrm{~cm}^{-3}$. For the chiral form $(12,6)$, they are $a=16.52 \AA$ and $\rho=1.40 \mathrm{~g} \mathrm{~cm}^{-3}$. More importantly, we determined the Young's modulus along the tube axis for triangular-packed SWNTs using the second derivatives of the potential energy. They are $Y_{(10,10)}=640.30 \mathrm{GPa}, Y_{(17,0)}=648.43 \mathrm{GPa}$, and $Y_{(12,6)}=$ 673.49 GPa, respectively. Normalized to carbon sheet these values are within a few per cent of the graphite bulk value.

\section{Vibrational modes and frequencies of SWNTs}

Using the second derivatives of the potential energy surface we calculated vibrational modes of a series of $(n, n)$ tubes. The uniform compression and highest in-plane modes are 
Table 3. Vibrational modes of $(10,10),(12,6)$, and $(17,0)$ single-wall nanotubes. Breathing mode $(B)$, shearing mode (S) and cyclops (C).

\begin{tabular}{|c|c|c|c|c|c|c|c|c|c|}
\hline \multirow[b]{2}{*}{ Mode } & \multicolumn{3}{|c|}{$(10,10)$} & \multicolumn{3}{|c|}{$(12,6)$} & \multicolumn{3}{|c|}{$(17,0)$} \\
\hline & $B$ & $S$ & $\mathrm{C}$ & $B$ & $S$ & C & $B$ & $S$ & C \\
\hline \multirow[t]{2}{*}{1} & & 111 & 242 & & 122 & 265 & & 113 & 247 \\
\hline & & 112 & 244 & & 122 & 265 & & 113 & 247 \\
\hline \multirow[t]{2}{*}{2} & 53 & 223 & 381 & 59 & 243 & 412 & 54 & 227 & 386 \\
\hline & 53 & 223 & 381 & 59 & 243 & 412 & 54 & 227 & 386 \\
\hline \multirow[t]{2}{*}{3} & 49 & 333 & 524 & 57 & 364 & 566 & 50 & 341 & 530 \\
\hline & 55 & 333 & 530 & 63 & 364 & 571 & 57 & 342 & 535 \\
\hline \multirow[t]{2}{*}{4} & 127 & 442 & 671 & 137 & 483 & 720 & 128 & 456 & 675 \\
\hline & 127 & 442 & 671 & 137 & 483 & 720 & 129 & 456 & 676 \\
\hline \multirow[t]{2}{*}{5} & 147 & 549 & 805 & 168 & 600 & 850 & 149 & 570 & 806 \\
\hline & 147 & 549 & 805 & 168 & 600 & 850 & 150 & 570 & 806 \\
\hline \multirow[t]{2}{*}{6} & 201 & 652 & 924 & 229 & 713 & 976 & 203 & 683 & 921 \\
\hline & 226 & 652 & 925 & 249 & 713 & 977 & 227 & 683 & 921 \\
\hline \multirow[t]{2}{*}{7} & 262 & 750 & & 299 & 819 & 1072 & 267 & 794 & 1017 \\
\hline & 272 & 750 & & 299 & 819 & 1072 & 267 & 794 & 1017 \\
\hline \multirow[t]{2}{*}{8} & 328 & 838 & & 360 & 912 & 1136 & 327 & 901 & 1091 \\
\hline & 330 & 838 & & 360 & 912 & 1136 & 328 & 901 & 1091 \\
\hline \multirow[t]{2}{*}{9} & 375 & 909 & & 396 & & 379 & 1002 & 1140 & \\
\hline & 381 & 909 & & 400 & & 385 & 1002 & 1140 & \\
\hline 10 & 402 & 939 & & 394 & & 434 & & & \\
\hline
\end{tabular}

listed in table 2 for $n=7$ to 13 . Among $(n, n)$ tubes we focused on the $(10,10)$ tube crystal. We also calculated the vibrational frequencies and modes for the zigzag $(17,0)$ and chiral $(12,6)$ tube crystals due to their comparable tube radius to $(10,10)$. We listed the breathing, shearing and cyclop modes for these equivalent radius nanotubes in table 3. These results may be used to differentiate chiral tubes with comparable diameters. The uniform compression mode is also shown in figure $7(a)$ and occurs at $186 \mathrm{~cm}^{-1}$ for $(10,10)$, which is exactly the same as the experimental frequency [24]. In table 3 the $\mathrm{B}$ denotes the breathing mode as displayed in figure $7(b), \mathrm{S}$ stands for shearing mode as in figure $7(c)$, and $\mathrm{C}$ stands for cyclops as in figure $7(d)$.

\section{Concluding remarks}

We presented a detailed study of structure, energetics and mechanical properties of SWNTs of varying size and chirality. We determined the stability domains for circular and collapsed cross section SWNTs. We reported the uniaxial strain-energy behavior of the SWNTs with crossover radius in the range of $-8 \%$ to $8 \%$ strain. The determined structure and lattice parameters for close packed $(10,10)$-like nanotubes are in close agreement with observations. We also determined vibrational modes and frequencies of bulk and isolated nanotubes using a highly accurate classical force field. Supplementary material is available at http://www.wag.caltech.edu

\section{Acknowledgments}

This research was funded by NASA (computational nanotechnology) and by NSF-GCAG (ASC 92-100368). The facilities of the MSC are also supported by grants from NSF
(CHE 95-22179), DOE-ASCI, ARO/DURIP, BP Chemical, ARO-MURI, Exxon, Seiko-Epson, Asahi Chemical, Beckman Institute, Chevron Petroleum Technology Co., Chevron Chemical Co., NASA/JPL, ONR, Avery Dennison, and Chevron Research Technology Co.

\section{References}

[1] Iijima S 1991 Helical microtubules of graphitic carbon Nature 354 56-8

[2] Iijima S and Ichlhashi T 1993 Single-shell carbon nanotubes of $1 \mathrm{~nm}$ diameter Nature 363 603-5

[3] Bethune D S, Kiang C H, Devries M S, Gorman G, Savoy R, Vazquez J and Beyers R 1993 Cobalt-catalyzed growth of carbon nanotubes with single-atomic-layer walls Nature 363 605-7

[4] Thess A et al 1996 Crystalline ropes of metallic carbon nanotubes Science 273 483-7

[5] Krotov Y A, Lee D-H and Louie S G 1997 Low-energy properties of $(n, n)$ carbon nanotubes Phys. Rev. Lett. 78 4245-8

[6] Tuzun R E, Noid D W, Sumpter B G and Merkle R C 1996 Dynamics of fluid-flow inside carbon nanotubes Nanotechnology 7 241-6

[7] Ihara S and Itoh S 1996 Molecular dynamics study of toroidal and helically coiled forms of graphitic carbon Surf. Rev. Lett. 3 827-34

[8] Chopra N G, Benedict L X, Crespi V H, Cohen M L, Louie S G and Zettl A 1996 Fully collapsed carbon nanotubes Nature 377 135-8

[9] Menon M, Richter E and Subbaswamy K R 1996 Structural and vibrational properties of fullerenes and nanotubes in a nonorthogonal tight-binding scheme $J$. Chem. Phys. 104 5875-82

[10] Hamada N, Sawada S and Oshiyama A 1992 New one-dimensional conductors-graphitic microtubules Phys. Rev. Lett. 78 1579-81

[11] Saito R, Fujita M, Dresselhaus G and Dresselhaus M S 1992 Electronic-structure of chiral graphene tubules Appl. Phys. Lett. 60 2204-6 
[12] Blase X, Benedict L X, Shirley E L and Louie S G 1994 Hybridization effects and metallicity in small radius carbon nanotubes Phys. Rev. Lett. 72 1878-81

[13] Robertson D H, Brenner D W and Mintmire J W 1992 Energetics of nanoscale graphitic tubules Phys. Rev. B 45 12592-5

[14] Yakobson B I, Campbell M P, Brabec C J and Bernholc J 1997 High strain rate fracture and c-chain unraveling in carbon nanotubes Comput. Mater. Sci. 8 341-8

[15] Richter E and Subbaswamy K R 1997 Theory of size dependent resonance raman scattering from carbon nanotubes Phys. Rev. Lett. 79 2738-41

[16] Iijima S, Brabec C, Maiti A and Bernholc J 1996 Structural flexibility of carbon nanotubes J. Chem. Phys. 104 2089-92

[17] Tersoff J and Ruoff R S 1994 Structural properties of carbon nanotube crystals Phys. Rev. Lett. 73 676-9

[18] Ruoff R S, Tersoff J, Lorents D C, Subramoney S and Chan B 1993 Radial deformation of carbon nanotubes by van der Waals forces Nature 364 514-16
[19] Guo Y J 1992 PhD Dissertation California Institute of Technology

[20] Guo Y J, Karasawa N and Goddard W A III 1991 Prediction of fullerene packing in C60 and C70 crystals Nature 351 464-7

[21] Gao G, Cagin T and Goddard W A III 1998 Position of K atoms in doped single walled carbon nanotube crystals Phys. Rev. Lett. 805056

[22] Lim K T, Brunett S, Iotov M, McClurg R B, Vaidehi N, Dasgupta S, Taylor S and Goddard W A III 1997 Molecular dynamics for very large systems on massively-parallel computers-MPSim program $J$. Comput. Chem. 18 501-21

[23] Landau L D and Lifshitz E M 1986 Elasticity Theory (Oxford: Pergamon)

[24] Rao A M et al 1997 Diameter-selective Raman scattering from vibrational modes in carbon nanotubes Science $\mathbf{2 7 5}$ $187-91$ 